sputum profiling enables non-invasive rapid sampling and evaluation, producing spectral signatures that can differentiate COPD status or predict exacerbation presence.

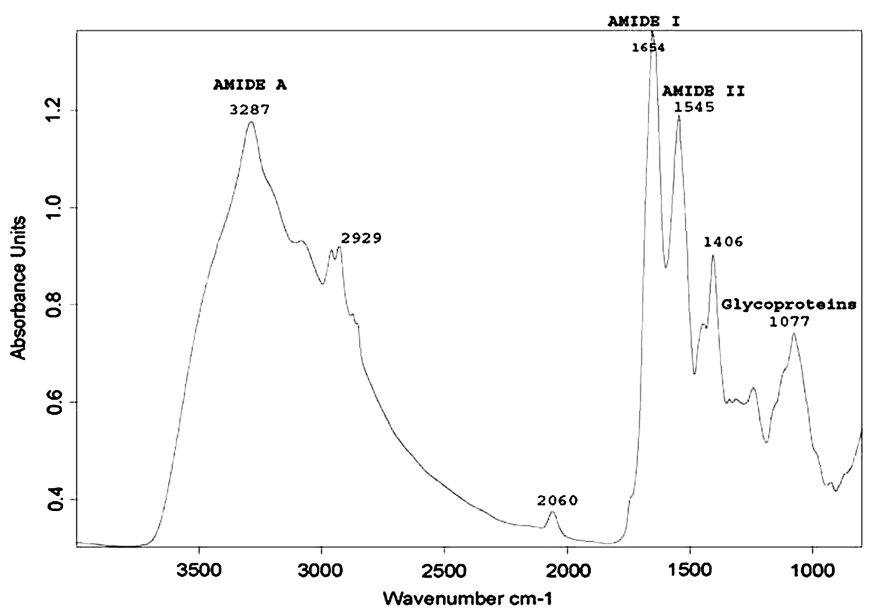

Abstract P111 Figure 1 Example of a typical IR absorption spectrum for COPD sputum.

\section{P112 ASSOCIATION OF SPUTUM PSEUDOMONAS AERUGINOSA (PSA) ISOLATION AND LENGTH OF HOSPITAL STAY IN PATIENTS WITH CHRONIC OBSTRUCTIVE PULMONARY DISEASE (COPD)}

doi:10.1136/thx.2010.150987.13

${ }^{1} \mathrm{~A}$ Ali, ${ }^{2} \mathrm{M}$ Bhattacharya, ${ }^{2} \mathrm{~J}$ Corcoran, ${ }^{3} \mathrm{~B}$ Chakraborty, ${ }^{1} \mathrm{~A}$ Thomas, ${ }^{4} \mathrm{M}$ Raza, ${ }^{1} \mathrm{R}$ Mukherjee. ${ }^{1}$ Department of Respiratory Medicine \& Physiology, Birmingham Heartlands Hospital, Bordesley Green East, Birmingham, UK; ${ }^{2}$ Department of Respiratory Medicine, Milton Keynes Hospital, Milton Keynes, UK; ${ }^{3}$ School of Mathematics, University of Birmingham, Birmingham, UK; ${ }^{4}$ Department of Microbiology, Milton Keynes Hospital, Milton Keynes, UK

Introduction and objectives A lot of effort and investment has been made to improve the length of stay (LOS) in COPD related admissions with variable success. It is known that numbers of hospital admissions and re-admissions have increased. Bacterial infections contribute to acute exacerbation of COPD (AECOPD) in $50 \%$ of cases; PsA is a probable pathogenic organism (PPM) causing acute or chronic infection in severe COPD patients. We set out to examine whether PsA in sputum influenced the length of stay as this PPM is not covered by the routinely used first line antibiotics. Method A retrospective audit was carried out examining the sputum culture results and LOS in all COPD admissions in an acute hospital from 1 January 2009 to 31 December 2009, patients were identified from coding (ICD: J44). Sputum cultures were attempted (and collected in all sputum producers) by a dedicated respiratory team as a routine for all COPD admissions throughout that period, which was established as part of service improvement well before the audit. Results were obtained from Pathology department database. Patients were divided into sputum producing group and nonproducers, Sputum producers were further divided into patients with positive sputum culture of PPM and non-pathogenic organisms. PsA growers were identified. Median LOS was calculated to be 5 days and all groups were compared with this duration as a standard for their LOS.

Results Total admissions with COPD were 332; 203 were sputum producers and 122 had bacterial isolates. There were 37 PsA growers. LOS was seen to be 5 or more days in: 121 sputum producers vs 47 non-producers, $\mathrm{p}=0.000046 .79$ PPM culture positive patients vs 42 without bacterial growth, $\mathrm{p}=0.067$; PsA growers vs. all admissions, $p=0.000744$, PsA vs all sputum producers $p=0.0126$, PsA vs all PPM, $\mathrm{p}=0.041$.

Conclusions PsA infection is associated with significantly longer LOS in hospital. All COPD patients should possibly be screened for PsA infection (and possibly underlying bronchiectasis) to inform the selection of antibiotics, which is likely to reduce LOS and may reduce re-admission rates.

\section{Estimated Distribution of Length of Stay}

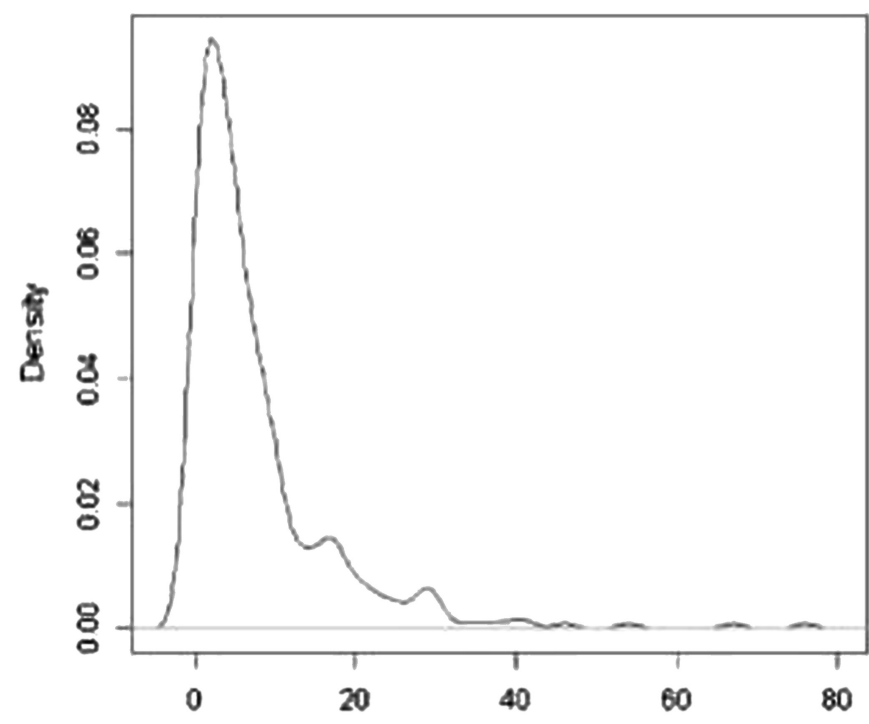

\section{P113 PROCALCITONIN (PCT) IS A SAFE AND RELIABLE BIOMARKER OF BACTERIAL INFECTION IN EXACERBATIONS OF COPD - SO WHY IS IT SO CHALLENGING TO INTRODUCE IT INTO A LARGE UK HOSPITAL?}

doi:10.1136/thx.2010.150987.14

L Burke, M Alhajji, N Todd, J S White. York Hospitals, York, UK

Background Antibiotics have only marginal efficacy in treating acute exacerbations of COPD (AECOPD). There is a large body of evidence that supports the use of PCT as a marker of bacterial infection. In York hospital patients with AECOPD account for a significant proportion of acute admissions and many are treated with antibiotics without strong evidence of a bacterial cause.

Objective To conduct a service evaluation of antibiotic prescribing following the introduction of PCT in patients with AECOPD. To observe attitudes towards and uptake of the test, and identify barriers to implementation.

Methods The evaluation ran from November 2009 to June 2010. Information on PCT was introduced to all physicians, and an algorithm for use was provided. Medical notes were reviewed from patients where PCT was requested. Evidence of documentation of PCT and whether it had been acted upon were recorded along with antibiotic use, length of stay and readmission within 1 month of discharge. Results 54 PCT tests were performed and 49 were included in the final analysis. $32(65 \%)$ of samples were below the cut-off for antibiotics, but were still prescribed in 11 cases. Of those above the threshold for treatment three did not receive antibiotics. PCT was documented and acted upon in only 12 (24\%) patients. Overall, antibiotics were used in $25(51 \%)$ patients. Two patients were readmitted with AECOPD within 1 month of discharge having been managed according to PCT results, with one receiving antibiotics. 24 $(49 \%)$ samples were not reported on the day they were taken.

Conclusion 11 RCTs from different countries enrolling over 3500 patients have demonstrated the feasibility and safety of PCT in 